\title{
"Evidence-based medicine" to support the surgical procedures we perform on patients with pelvic organ prolapse
}

\author{
M. M. Karram
}

Received: 30 April 2009/Accepted: 4 May 2009/Published online: 15 May 2009

(C) The International Urogynecological Association 2009

While no one would argue that evidence is certainly the cornerstone of science, the term "evidence-based medicine" is commonly utilized by academicians, clinicians, researchers, and policy makers. The goal of an "evidence-based" health-care system is to provide quality health care based on a consensus within a subspecialty of standards of clinical practice that lead to diagnostic and treatment guidelines. "Evidence-based medicine" is defined as the conscientious, explicit, and judicious use of current best evidence in making decisions about the care of individual patients. The decisions are based on three factors:

1. Best available external evidence

2. Individual clinical expertise and judgment

3. Patient preferences

Application of "evidence-based medicine" comes under several names such as "clinical practice guidelines," "clinical algorithms," "clinical policies," and "clinical pathways." The process of using scientific studies to form clinical guidelines that direct clinical decision making and patient care is certainly more straightforward in certain conditions than in others. For example, it is reasonable to create a standard algorithm to evaluate and treat urinary incontinence. However, when we speak about surgery for pelvic organ prolapse this becomes a much more difficult and controversial subject. The goals of any surgery for pelvic organ prolapse should be to correct the anatomic problem as well as maintain or restore visceral and sexual function.

M. M. Karram $(\bowtie)$

Obstetrics and Gynecology, Good Samaritan Hospital,

3219 Clifton Avenue, Suite 100,

Cincinnati, OH 45220-2489, USA

e-mail: iujournal.office@gmail.com
This, however, becomes a very difficult task as our understanding of the relationship between anatomic descent and functional derangements continues to be very poor. For example, four women of the exact same age, menopausal status, and parity with what appears to be the same anatomic picture (descent of the anterior vaginal wall to just outside of the hymen when they strain in a supine position) could present with an entire array of lower urinary tract dysfunction. Patient number 1 may have no lower urinary tract symptoms; patient number 2 may suffer from symptoms of overactive bladder, patient number 3 may have stress incontinence, and patient number 4 may have to reduce her prolapse to efficiently empty her bladder. Scientific evidence to explain such a wide array of functional derangements in what appears to be the same anatomic picture is lacking. So, as pelvic floor surgeons take steps to practice "evidence-based medicine" they need to formulate a clear clinical question that defines the patients' problems, i.e. is the patient's problem more functional, more anatomic, or both, and what is the likelihood of surgical intervention providing a long-term correction of the problem. Add to this the fact that the technical aspects of our procedures are not standardized, and much controversy continues to exist regarding the best procedure for any specific presentation.

While most of us would agree that the primary anatomic goal of a procedure for prolapse should be to achieve high, durable support of the apex without distorting the vaginal axis, how best to accomplish this remains very controversial [1].

Also, we certainly cannot forget that the patient must significantly be involved in your decision process. As new materials, new techniques, and new devices are developed, the surgeon must fully disclose his experience 
with them as well as the results of the procedure or material that may be utilized.

It is also important to consider how complication rates and the technical expertise and surgical experience of the person performing the procedure should play into "evidence-based medicine". As time goes on, procedures are modified in the hope of improving outcomes. We, as a subspecialty, need to determine which changes are of enough significance to require objective studies versus those changes that are subtle and are obviously, to the operator, advantageous over previously described techniques.

"Evidence-based medicine" was first developed in the 1980s at McMaster University School of Health Sciences in Ontario, Canada. It is a clinical learning strategy that teaches physicians to turn a clinical problem into a question that can be answered by a systemic review and evaluation of the most current results from published studies, and then applied to a particular clinical problem [2]. The process of using scientific studies to form clinical guidelines that direct clinical decision making and patient care is still evolving [3].

In conclusion, surgeons will continue to perform procedures that they feel they do best and are most suited for their patients. Industry will continue to develop and promote new materials and devices in the hope of simplifying procedures and improving outcomes, as they have realized that this is a very big market. As time goes on our menu options will only increase. While ideally we would love level one evidence to support what we do, it is unrealistic that this will be available in a timely fashion. In the interim we must hope that surgeons will be forthcoming in honestly reporting their results and complications with whatever intervention they are performing.

\section{References}

1. Diwadkar GB, Barber M, Fenner B, Maher C, Jelovsik EJ (2009) Complications and reoperation rates after apical vaginal prolapse surgical repair. A systematic review. Obstet Gynecol 113:367-373

2. Rosenberg W, Donald A (1995) Evidence based medicine: an approach to clinical problem solving. Br Med J 310:1122-1126

3. McKivvon A (1999) Secondary publications: clinical practice guidelines. In: PDQ evidence based principles and practice. Decker, London, p 157 